\title{
INFLUENCE OF GENDER ON MUSCLE ACTIVITY PATTERNS DURING NORMAL AND FAST WALKING
}

\author{
Manvinder Kaur ${ }^{1}$, Sateesh Reddy Avutu ${ }^{1}$, Dinesh Bhatia*1, Suresh Verma ${ }^{2}$ \\ ${ }^{1}$ Biomedical Engineering Department, North Eastern Hill University, \\ Shillong-793022, Meghalaya, India \\ ${ }^{2}$ Department of Mechanical Engineering, Deenbandhu Chhotu Ram University of Science \\ \& Technology, Murthal-131039, Haryana, India
}

\begin{abstract}
Electromyography (EMG) signals are often described as electrical manifestation of neuromuscular activation associated with the muscles. These signals are commonly utilized as principal input signals to control several prosthetic devices such as prosthetic hands, arm, lower limbs, and exoskeleton robots as well as in designing of rehabilitation and assistive devices. It is well proven that EMG signals vary among subjects and gender is one of the major factors that play a significant role in this variation. This study detects the possible gender differences by measuring changes in the EMG activity during different phases of human walking by acquiring the surface EMG signals from Gluteus Maximus, Hamstrings (biceps femoris), Quadriceps (rectus femoris) and Soleus muscles of the leg with the healthy subjects walking barefoot at two paces-normal and fast. The statistical analysis of the results showed no gender differences at normal speed of walking but when speed of walking changed; it showed clear differences in the behavior of these muscles. The results from this study would aid in designing closed loop control strategy for designing a smart functional electrical stimulator (FES) which is the larger goal of this research.
\end{abstract}

\section{KEYWORDS}

Electromyography, Walking, gender

\section{INTRODUCTION}

Analysis of human walking is a significant area of research in which measurements of muscle activity using EMG signals have often been used. As per the established literature, EMG signal has proved to be a significant tool in the analysis of gait related disorders [1,2]. It is commonly employed as principal input information to the controller for utilization in wide range of medical applications ranging from powered assistive devices, bio-amplifiers to exoskeletons and rehabilitation devices such as functional electrical stimulator (FES) $[11,12,18]$.

The EMG signals normally show tendency to vary among individuals. It is a proven fact that no two individuals have same gait pattern and EMG variations may be observed for performing the similar task within the same individual, making it unreliable at times [1,2]. Nevertheless it is still utilized by different research group(s) worldwide working in the above mentioned areas for providing innumerable healthcare benefits. When designing closed loop strategy for FES devices, characteristics of EMG must be carefully considered for providing proper input information to the controller. Gender has been found to play a significant role and is one of the important factors that affect EMG activity in different motor tasks [7, 10]. Murray et al. found that the amplitude of normalized EMG activity decreased as walking speed decreased in normal women subjects [17]. M.C. Chiu etc. all. Found that increased walking speed caused significant increase in the muscle activities of lumbar erector spinae, biceps femoris, and medial gastrocnemius, lumbar motion, as well as the vertical ground reaction force in the loading response and mid-stance phases of the

DOI: 10.5121/ijbes.2016.3304 
human gait cycle [5]. E.S. Chumanov et. al. findings indicated that females display greater nonsagittal motion [6]. Nardo et. al. reported propensity of females for a more complex recruitment of the muscles such as gluteus and tibialis during gait [10].

Based on these studies, the main objective of this paper was to evaluate possible gender related differences in the EMG activity of four significant lower limb muscles Gluteus Maximus, Soleus, Quadriceps (rectus femoris) and Hamstring (biceps femoris) for both the legs. Gluteus Maximus, Quadriceps and Hamstring muscle group are considered to be large prime movers during walking along with soleus being utilized for plantar flexion of the ankle, from [3] we can conclude that Soleus is more active muscle compared with the gastronomies during walking. Rectus femoris is opted from the quadriceps muscle group because it is a hip flexor muscles, as it attaches to the Ilium which is a crucial indicator during walking or running. It swings the leg forward into the ensuing step during walking $[15,19]$. Biceps femoris is selected from the hamstring muscle group because it is considered to be major antagonist muscle to the quadriceps during the deceleration of knee extension during walking $[15,19]$.

We hypothesized that significant gender related differences would be found during normal and fast walking, in this paper $80-$ steps/min is considered as a slow walking and $120 \mathrm{steps} / \mathrm{min}$ is considered as a fast walking. The results of this analysis would provide significant information on gender-related differences thereby suggesting the researchers and clinicians with an alternative approach for males and females when designing closed loop strategy for prosthetic devices by employing EMG signals.

\section{METHODOLOGY}

The methodology followed for carrying out the above study is shown schematically in Figure1.

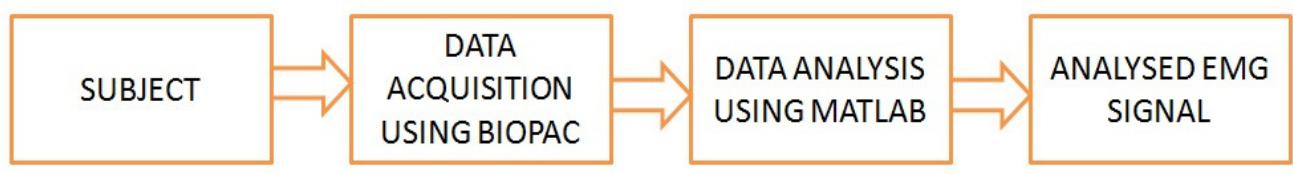

\section{A. Participants}

Figure -1

Twenty healthy adult volunteers were recruited. 10 male, 10 Female, mean age for male $23 \pm 2$ year, for Female $22 \pm 2$ year, Mean height for male $164 \pm 5 \mathrm{in} \mathrm{cm}$, for female $152 \pm 3$ in $\mathrm{cm}$ and mean weight for male $68 \pm 6$ in $\mathrm{Kg}$, for female $54 \pm 5$ in $\mathrm{kg}$. The Mean $( \pm \mathrm{SD})$ age, height and weight are shown in Table I.

\begin{tabular}{|c|c|c|c|}
\hline Category & $\begin{array}{c}\text { Age in Years } \\
(\text { Mean } \pm \text { SD })\end{array}$ & $\begin{array}{c}\text { Height }(\mathrm{cm}) \\
(\text { Mean } \pm \text { SD })\end{array}$ & $\begin{array}{c}\text { Weight }(\mathrm{Kg}) \\
(\text { Mean } \pm \text { SD })\end{array}$ \\
\hline Female & $22 \pm 2$ & $152 \pm 3$ & $54 \pm 5$ \\
\hline Male & $23 \pm 2$ & $164 \pm 5$ & $68 \pm 6$ \\
\hline
\end{tabular}

TABle I: Characterstics Of FemaleAnd MALE SubJeCtS

EMG activity was recorded from the selected muscles of both the left and right lower limbs of all subjects during barefoot gait. Inclusion criteria excluded subjects having any medical conditions/ contractures/ deformities in the joint or suffering from skin condition which might impede the fixation of the electrodes on the body surface. Before participating in the study each participant was explained about the purpose and protocol to be followed for the study. The participants 
agreed to provide written informed consent/ patient informed consent (PIC) form as per requirement of the Ethics Committee before participating in the study.

\section{B. Instrumentation}

EMG Signals were acquired using Multi-Channel Wireless EMG BIOPAC Inc. (CMRR: 110dB at 50/60 Hz and Gain: 5-50,000, Input Impedance: $2 \mathrm{M} \Omega$ ) available in the laboratory for the study. The skin was rubbed with cotton containing alcohol to minimize the skin impedance, thereby improving signal acquisition. Disposable Circular electrodes (44 x $32 \times 1 \mathrm{~mm})$ were placed on the subjects for the above muscles, with respect to longitudinal location of the sensor on the muscle and halfway the distal motor endplate zone and the distal tendon, with respect to the transversal location of the electrode [20]. The subject preparation was carried out following standard protocols [14] for placing electrodes on subject's pre-identified muscles to acquire EMG simultaneously from both the legs. In order to avoid the cross talk between corresponding muscle fibers, we placed the electrodes around the optimal sensor location directed parallel to the muscle fibers with an inter-electrode distance of $20 \mathrm{~mm}$.

\section{Data Acquisition}

The study is carried out for each subject in a quiet laboratory room away from the main entrance having very few visitors. The subject and research team cannot interact once the experiment has started. Test duration is about one hour per subject including the time required for mounting the devices to the subject. In order to prevent contamination of the signal, the cables of the sensor are fixed to the subject's body using the straps. Sampling rate during acquisition was set to $2000 \mathrm{~Hz}$ as per Nyquist criteria Schematic view of experimental environment is shown in Figure 2.

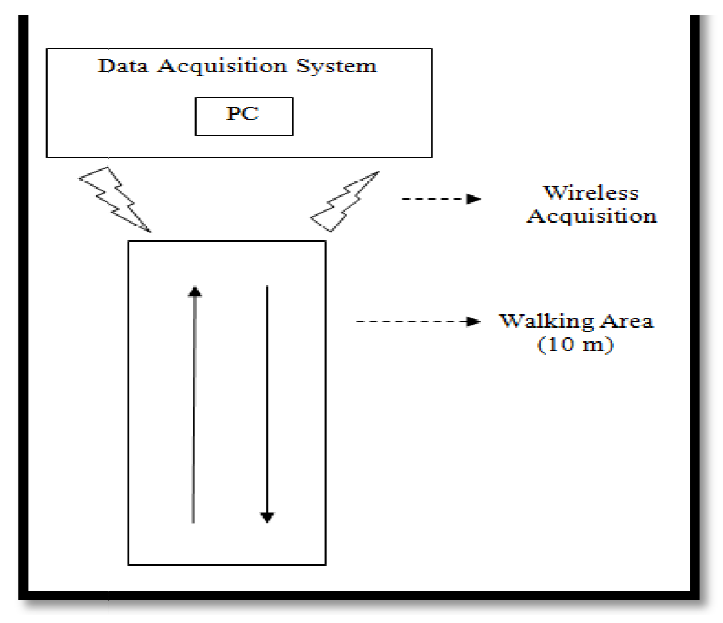

Figure 2: Schematic view of experimental environment

Maximum voluntary contraction (MVC) tests were conducted on the muscles for intra subject comparisons by applying the resistance manually to resist the subject's movement. For the Gluteus Maximus muscle, the subject was made to lie on prone position and the MVC test was performed both in extended and flexed knee position with slightly outward rotated legs and for Soleus muscle the subject was asked to sit in up-right position to perform unilateral Plantar flexion. In case of Quadriceps muscle, the subject was asked to sit in upright position to perform knee flexion position and for Hamstring muscle the subject was made to lie on prone position to perform unilateral knee Flexion. Four sets of voluntary data were acquired, with the subjects barefoot walking in the selected area at an average speed 80steps/min for normal and 120 steps/min for fast walking. It was determined to ask the subjects to walk at different speeds, as it 
is usually observed that individuals usually walk at either slow or fast pace daily on different occasions as per their task requirements. The collected data was stored using Acknowledge 4.3 software available with the data recording system.

\section{Data Processing}

For processing of EMG signal, normalization of the EMG signal acquired from each subject with its isometric MVC. Notch filter was then applied in order to remove $50 \mathrm{~Hz}$ noise interference from the signal. Subsequently, Wavelet transform (WF) was performed for removing baseline drift in the signal. The WF technique permits time-frequency representation of the signal thereby allowing inspection of different signal waveforms at different scales and resolution. Cascaded low-pass $(20 \mathrm{~Hz})$ and a high-pass filter $(450 \mathrm{~Hz})$ were then applied in order to remove other noise sources from the signal. The raw EMG signals acquired from the subjects were quantified with the help of MATLAB. The Signal Processing applied onto the raw EMG signals is explained and shown in Figure 3.

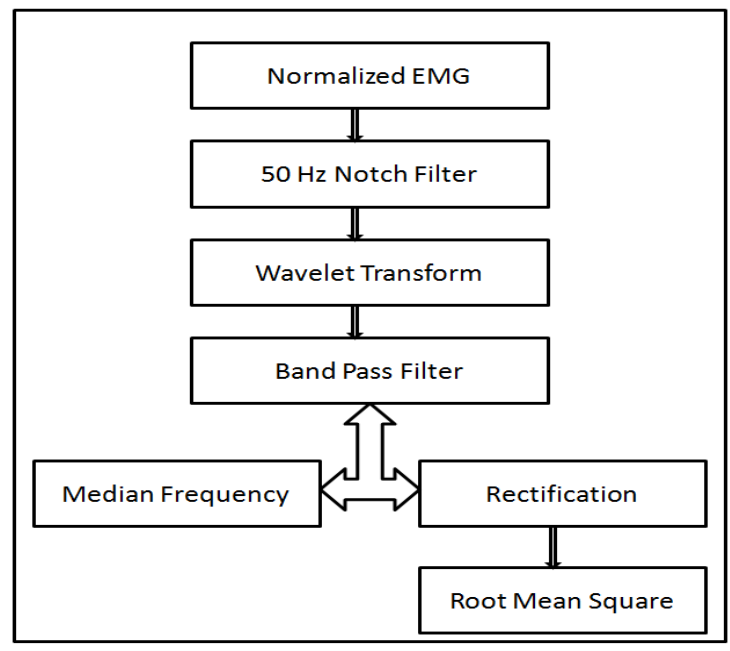

Figure 3: Signal Processing Steps

All four recorded data sets for each subject were analyzed to calculate two major parameters from the filtered EMG: Root Mean Square (RMS) and Median Frequency (MDF), Since the MDF decreases with an increase in EMG signal amplitude which is the strong indication of the muscle fatigue. The filtered EMG signals were rectified in order to calculate the RMS values. The mean values of all the walking trials (normal and fast pace) were identified and averaged to derive the mean values for these two parameters.

\section{1) Root Mean Square (RMS)}

RMS is the square root of the arithmetic mean of the squares of the set of values. Let $X_{t}$ represent the rectified EMG signal then RMS value is represented by equation 1

$$
\mathrm{RMS}=\sqrt{\frac{1}{T-t} \sum_{t}^{T}\left(\mathrm{X}_{\mathrm{t}}\right)^{2}}
$$

Where, $\mathrm{X}_{\mathrm{t}}$ is the rectified signal and $\mathrm{T}, \mathrm{t}$ is the two time intervals at which signal acquisition takes place. It is most frequently used parameter for the EMG analysis because during muscle 
contractions, it reflects the level of physiological activity in the motor unit. It is considered to be the most meaningful EMG analysis technique, since it gives a measure of the mean power of the signal [9].

\section{2) Median Frequency (MDF)}

MDF is a frequency at which the EMG power spectrum is divided into two regions with equal amplitude [9] as shown in equation 2:

$$
\underset{j=1}{\operatorname{MDF}} P_{j}=\sum_{j=M D F}^{M} P_{j}=1 / 2 \sum_{j=1}^{M} P_{j}
$$

Where, $P_{j}=E M G$ power spectrum at the frequency $j$ and $M=$ length of frequency

It helps the clinician to focus on injury prevention strategies, especially during the restorative phase of rehabilitation [14].

\section{E. Statistical Analysis}

For detailed analysis, statistical validation is done by applying one way ANOVA that depicted the influence of gender is statistically significant during variations in walking speed. Least Significant differences (LSDs) among the categories defined were then conducted using Post hoc analysis in order to have multiple comparisons between the categories using SPSS software (IBM). The level of significance was set to $\mathrm{p}<0.05$.

\section{RESULT}

The data is divided into four categories namely female with normal walk (FNW), female with fast walk (FFW), male with normal walk (MNW) and male with fast walk (MFW). According to Table II, the comparison between the categories MNW and MFW and FFW and MFW were found to be statistically significant at 0.05 level. Other categories mentioned in table such as MNW with FFW or FNW with MFW were found to be invalid. All the other groups were found to be statistically insignificant at 0.05 level. The results so obtained from the comparison through statistical analysis using LSD ignoring the ANOVA table are shown in Table II. 
International Journal of Biomedical Engineering and Science (IJBES), Vol. 3, No. 3, July 2016

TABLE II: LEAST SIGNIFICANT DIFFFRENCES BETWEEN THE GROUPS OF RMS AND MDF VALUES

Trace titles RMS_G_R, RMS_S_R, RMS_Q_R, RMS_H_R, RMS_G_L, RMS_S_L, RMS_Q_L and RMS_H_L refer to the RMS value obtained from Gluteus,

\begin{tabular}{|c|c|c|c|c|c|c|c|}
\hline $\begin{array}{l}\text { Dependent } \\
\text { Variable }\end{array}$ & Category I & Category J & Sig. & $\begin{array}{c}\text { Dependent } \\
\text { Variable }\end{array}$ & Category I & Category $\mathbf{J}$ & Sig. \\
\hline \multirow{6}{*}{ RMS_G_R } & \multirow{3}{*}{ FNW } & FFW & 0.307 & \multirow{6}{*}{ MDF_G_R } & \multirow{3}{*}{ FNW } & FFW & 0.965 \\
\hline & & MNW & 0.591 & & & MNW & 0.001 \\
\hline & & MFW & 0.11 & & & MFW & 0.039 \\
\hline & \multirow{2}{*}{ FFW } & MNW & 0.644 & & \multirow{2}{*}{ FFW } & MNW & 0.001 \\
\hline & & MFW & 0.532 & & & MFW & 0.035 \\
\hline & MNW & MFW & 0.292 & & MNW & MFW & 0.157 \\
\hline \multirow{6}{*}{ RMS_S_R } & \multirow{3}{*}{ FNW } & FFW & 0.004 & & \multirow{3}{*}{ FNW } & FFW & 0.981 \\
\hline & & MNW & 0.636 & & & MNW & 0.007 \\
\hline & & MFW & 0.003 & & & MFW & 0.019 \\
\hline & \multirow{2}{*}{ FFW } & MNW & 0.015 & NIDIS_S_R & \multirow{2}{*}{ FFW } & MNW & 0.007 \\
\hline & & MFW & 0.915 & & & MFW & 0.02 \\
\hline & MNW & MFW & 0.013 & & MNW & MFW & 0.699 \\
\hline \multirow{6}{*}{ RMS_Q_R } & \multirow{3}{*}{ FNW } & FFW & 0.148 & & \multirow{3}{*}{ FNW } & FFW & 0.716 \\
\hline & & MNW & 0.198 & & & MNW & 0.451 \\
\hline & & MFW & 0.203 & $M D F \cap \mathbf{P}$ & & MFW & 0.318 \\
\hline & \multirow{2}{*}{ FFW } & MNW & 0.009 & 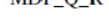 & \multirow{2}{*}{ FFW } & MNW & 0.688 \\
\hline & & MFW & 0.01 & & & MFW & 0.516 \\
\hline & MNW & MFW & 0.987 & & MNW & MFW & 0.808 \\
\hline \multirow{6}{*}{ RMS_H_R } & \multirow{3}{*}{ FNW } & FFW & 0.241 & & \multirow{3}{*}{ FNW } & FFW & 0.6 \\
\hline & & MNW & 0.732 & & & MNW & 0.018 \\
\hline & & MFW & 0.008 & MDE H & & MFW & 0.099 \\
\hline & \multirow{2}{*}{ FFW } & MNW & 0.421 & NIDIIIIN & \multirow{2}{*}{ FFW } & MNW & 0.005 \\
\hline & & MFW & 0.106 & & & MFW & 0.034 \\
\hline & MNW & MFW & 0.021 & & MNW & MFW & 0.444 \\
\hline & & FFW & 0.266 & & & FFW & 0.966 \\
\hline & FNW & MNW & 0.268 & & FNW & MNW & 0.021 \\
\hline PMS G I & & MFW & 0.004 & $M D E G$, & & MFW & 0.081 \\
\hline KIVIS_U_L & FFW & MNW & 0.981 & MDF_U_L & FFW & MNW & 0.023 \\
\hline & & MFW & 0.05 & & & MFW & 0.088 \\
\hline & MNW & MFW & 0.059 & & MNW & MFW & 0.543 \\
\hline & & FFW & 0.357 & & & FFW & 0.999 \\
\hline & FNW & MNW & 0.261 & & FNW & MNW & 0.059 \\
\hline RMS S I & & MFW & 0.357 & MDE S J & & MFW & 0.062 \\
\hline KMS_S_L & FFW & MNW & 0.815 & MDI_S_L & FFW & MNW & 0.059 \\
\hline & $117 \mathrm{w}$ & MFW & 0.006 & & & MFW & 0.062 \\
\hline & MNW & MFW & 0.013 & & MNW & MFW & 0.983 \\
\hline & & FFW & 0.272 & & & FFW & 0.924 \\
\hline & FNW & MNW & 0.298 & & FNW & MNW & 0.583 \\
\hline RMS 0 I & & MFW & 0.307 & MDF 0 & & MFW & 0.562 \\
\hline & FFW & MNW & 0.039 & $1001-2-\mathrm{E}$ & FEW & MNW & 0.522 \\
\hline & FFW & MFW & 0.041 & & FFW & MFW & 0.502 \\
\hline & MNW & MFW & 0.985 & & MNW & MFW & 0.976 \\
\hline & & FFW & 0.179 & & & FFW & 0.857 \\
\hline & FNW & MNW & 0.292 & & FNW & MNW & 0.009 \\
\hline RMS H I & & MFW & 0.004 & MDF $\mathrm{H}$ & & MFW & 0.013 \\
\hline Kiven_L & FFW & MNW & 0.794 & MIDF_H_L & FEW & MNW & 0.005 \\
\hline & FTw & MFW & 0.084 & & FrW & MFW & 0.009 \\
\hline & MNW & MFW & 0.055 & & MNW & MFW & 0.861 \\
\hline
\end{tabular}

Soleus, Quadriceps and Hamstrings respectively for right and left leg. Similarly, the trace titles MDF_G_R, MDF_S_R, MDF_O R, MDF_H_R, MDF G L

MDF_S_L, MDF_Q_L and MDF_H_L refer to the MDF values obtained from Gluteus, Soleus, Quadriceps and Hamstrings respectively for right and left leg.

Further analysis demonstrates the processed EMG data through the results of descriptive statistics for the RMS and MDF values is depicted as shown in Figure 4. and Figure 5. From the figures, it can be observed that the mean RMS value of the signal amplitude tends to increase from normal to fast walking for all the muscles of both the limbs, which can be attributed to increase in motor activity and recruitment of more fibers with increase in speed of movement. MDF value follows an inverse pattern which is opposite to the variations in time domain feature of the EMG signal. It decreases when walking speed changes from normal to fast indicating development of muscle fatigue in fibers. 


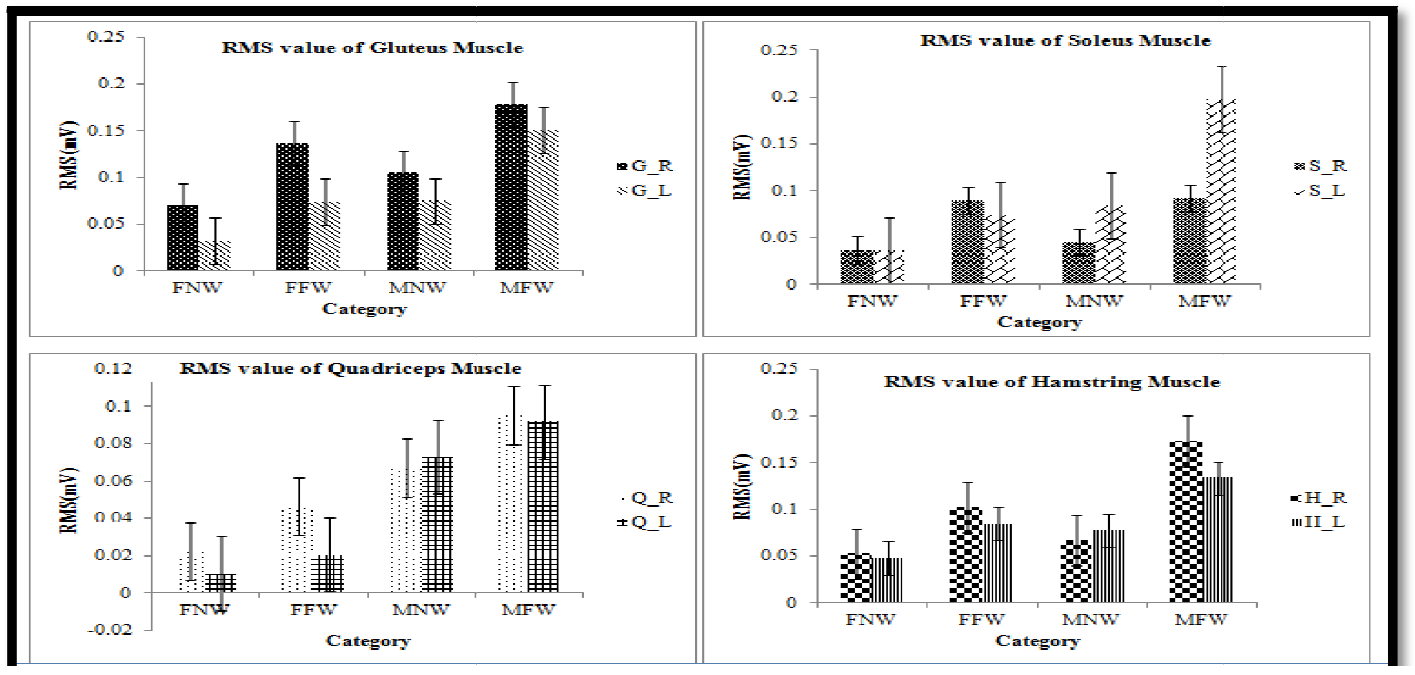

Figure 4: Comparison of RMS values on basis of gender

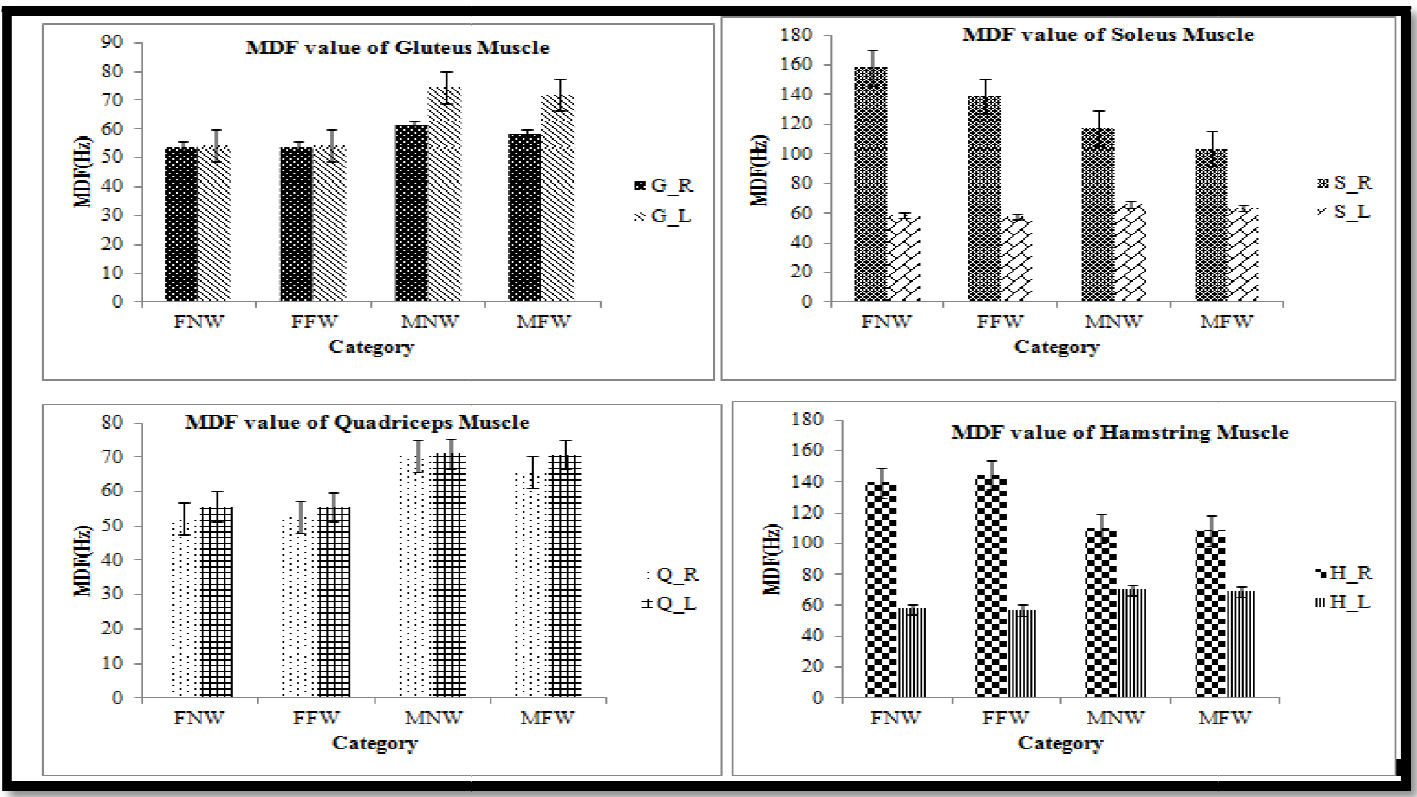

Figure 5: Comparison of MDF values on basis of gender

\section{DISCUSSION OF FINDINGS}

The paper evaluated the gender differences on the major lower limbs muscles namely, Gluteus Maximus, Hamstring (biceps femoris), Quadriceps (rectus femoris) and Soleus with the help of surface EMG during barefoot walking. Quantification of EMG signal was done using both time and frequency domain parameters. For time domain, RMS was chosen to assess the level of muscle activity, since it is not affected by the superposition of action potential on motor units [9]. For frequency domain, parameter opted for study was median frequency because it is sensitive to the physiological processes that occur within the muscles during sustained contractions. The statistical analysis showed no gender differences during normal walking but when speed of 
walking changed from normal to fast, gender differences were prominent and found to be significant for all the muscles under study. These results are partly in line with our hypothesis stated above.

It is observed through the results of descriptive statistics that RMS value of males is much higher than that of females RMS values obtained for Gluteus, Quadriceps (rectus femoris) and Hamstring (biceps femoris) muscles shows that right leg is predominant over left leg for both males and females. However, the Soleus muscle of male left leg is predominant over right leg. This may due to the fact that during walking soleus activity is greatest on left side for most male subjects under study.

MDF values also indicate the propensity of males over females to show changes in the muscle activity patterns when the speed of walking changed. For Gluteus and Quadriceps muscles, left leg is predominant over right leg for both males and females but for Soleus and Hamstrings muscles, the right leg is predominant over left leg. This may be due to reasons such as the modulation in recruitment affecting firing rate of the motor units and synchronization of the EMG signal. Moreover, the variations in the fiber diameters and due to gender differences may also be one of the causes for such changes observed $[4,8,13]$. For Gluteus and Quadriceps muscles, the MDF value is much higher in males than in females.

Understanding the above analysis, it is evident that in the healthy males significant changes are shown in MDF and RMS values when walking speed changes from normal to fast. No such changes were shown in females when walking speed changes. This shows the propensity of males over females for muscle activity patterns of gluteus, Soleus, rectus femoris and biceps femoris which are contrasting to the results reported by earlier researchers $[6,10]$.

There are limitations to the current study that can be further investigated. The lack of kinematic and kinetic data means that confounding variables may be present. The group of participants observed here consisted of young participants and these findings may not be generalized to older populations. Despite these issues, this would also help to identify the normal ranges of EMG variations for a local adult population under study for developing EMG based controls in wide range of clinical and engineering applications in near future.

\section{CONCLUSION}

The final are the conclusive remarks of the study:

1) Root Mean Square (RMS) increases when pace of walking changed from normal to fast

2) Median Frequency (MDF) value decreases when pace of walking changed from normal to fast.

3) Statistical analysis showed significant variation in the MDF and RMS values when walking speed changes from normal to fast in healthy males.

4) No significant changes were shown in females when walking speed changes.

5) The statistical analysis showed no significant changes in gender differences were found during normal walking but when speed of walking changed from normal to fast, gender differences were found to be significant for all the above muscles.

6) Overall propensity of males is found to be superior over females for muscle activity patterns of gluteus, Soleus, rectus femoris and biceps femoris.

This information suggests considering a separate approach for males and females when designing a closed loop controller strategy for rehabilitation devices such as FES, prosthetic limbs using electromyography (EMG) signals. 


\section{ACKNOWLEDGMENT}

This work is supported by funding received (Ref: IDP/MED/2010/27; 2012) from the Instrument Development Program of the Department of Science and Technology (DST), Government of India, New Delhi. The authors also acknowledge the support of the doctors from the Physical Medicine Rehabilitation Department, AIIMS, Delhi involved in the above project.

\section{SUBMISSION STATEMENT}

We represent that this submission is original work, and is not under consideration for publication with any other journal.

\section{REFERENCES}

1. Bansal G, Bhatia D, Joshi D, Anand S, Tewari RP. Coordination between lower limb muscles in different locomotion activities. International Journal of Biomedical Engineering and Technology (IJBET), 6(2):129-141, 2011.

2. Basmajian JV, Deluca, CJ. Muscles alive: their functions revealed by electromyography. Baltimore: Williams \& Wilkins, 5th Ed., 19-64, 1985.

3. Bhatia D, Bansal G, Tewari RP, Shukla KK. Study the role of muscles under different loading conditions using EMG analysis of lower extremities. Advances in Applied Science Research, 1(3): 118-128,2010.

4. Buxbaum J, Mylinski N, Parente FR. Surface EMG reliability using spectral analysis. Journal of Oral Rehabilitation, 23(11):771-775, 1996.

5. Chiu MC, Wang MJ. The effect of gait speed and gender on perceived exertion, muscle activity, joint motion of lower extremity, ground reaction force and heart rate during normal walking. Gait Posture, 25:385-392, 2007.

6. Chumanov ES, Wall-Scheffler C, Heiderscheit BC. Gender differences in walking and running on level and inclined surfaces. Clin. Biomech. (Bristol, Avon), 23:1260-1268, 2008

7. Cowan SM, Crossley KM. Does gender influence neuromotor control of the knee and hip. Journal of Electromyography and Kinesiology, 19(2):276-282, 2009.

8. De Luca CJ, Sabbahi MA, Roy SH. Median frequency of the myoelectric signal:Effects of hand dominance. Eur J Appl Physiol Occup Physiol.,55(5):457-64,1986.

9. De Luca CJ. Surface electromyography: detection and recording. DelSys Inc. Copyrighted 2002.

10. Di Nardo F., Mengarelli A., Maranesi E., Burattini L. Influence of gender on the myoelectric signal of shank muscles. IEEE-MESA, 1-4, 2014.

11. Farry KA, Walker ID and Baraniuk RG. Myoelectric Teleoperation of a Complex Robotic Hand. IEEE Trans Rob Autom, 12(5):775-788, 1996.

12. He H., Kiguchi K. and Horikawa E. A Study on Lower-Limb Muscle Activities during Daily Lower-Limb Motions. Int J Bioelectromagn, 9 (2):79-84, 2007.

13. Inman V, Ralston H, Todd F. Human walking. Baltimore: Williams \& Wilkins, 1981.

14. Konard P. The ABC of EMG: A Practical Introduction to Kinesiological Electromyography. Noraxon Inc. USA, 1, April 2005.

15. Mansour JM, Pereira JM. Quantitative functional anatomy of the lower limb with application to human gait. Journal of Biomechanics, 20:51-58, 1987.

16. McMahon TA, Cheng GC. The mechanics of running-how does stiffness couple with speed. Journal of Biomechanics , 23:S65-S78, 1990.

17. Murray MP, Mollinger LA, Gardner GM and Sepic SB. Kinematic and EMG patterns during slow, free, and fast walking. Journal of Orthopaedic Research, 2(3):272-280, 1984

18. Perry JC, Rosen J. Upper-Limb Powered Exoskeleton Design. IEEE/ASME Transaction on Mechatronics, 12(4): 408-417, 2007

19. Saladin KS. Anatomy \& Physiology: The Unity of Form and Function. McGraw-Hill, New York, 6th ed., 2007.

20. Seniam: European recommendations for surface electromyography. Roessingh Research and Development, Enschede, Holland, 1999. 